\title{
Unbroken flavour symmetries vs lepton masses and mixings
}

\author{
Yakefu Reyimuaji \\ SISSA/ISAS and INFN, Via Bonomea 265, 34136 Trieste, Italy \\ ICTP, Strada Costiera 11, 34151 Trieste, Italy \\ E-mail: yreyimuaesissa.it

\section{Andrea Romanino*} \\ SISSA/ISAS and INFN, Via Bonomea 265, 34136 Trieste, Italy \\ ICTP, Strada Costiera 11, 34151 Trieste, Italy \\ E-mail: romanino@sissa.it
}

\begin{abstract}
Assuming that light neutrino masses are described by the Weinberg operator and constrained by a flavour symmetry, we identify all flavour groups and representations providing an approximate description of lepton masses and mixings in the symmetric limit. It turns out that the neutrinos are always either anarchical or have an inverted hierarchical spectrum. We then investigate whether the flavour theory predictions obtained within the Weinberg operator description are equivalent to those obtained within the full UV theory, using type I seesaw as a prototypical example. That is not always the case, and the conditions for the equivalence are found. If the hint of a normal hierarchical neutrino spectrum were confirmed, we would then conclude that either the symmetry breaking effects play a primary role in the understanding of neutrino flavour observables, or the UV flavour theory does not provide the same predictions as the effective one.
\end{abstract}

Neutrino Oscillation Workshop (NOW2018)

9 - 16 September, 2018

Rosa Marina (Ostuni, Brindisi, Italy)

* Speaker. 
Flavour symmetries [1-15] have long represented one of the most popular attempts in the effort to understand the pattern of fermion masses and mixings. In order to provide an accurate description of the flavour observables, the flavour symmetry must be (spontaneously) broken. Depending on the model, the role of the flavour breaking can be substantially different. In some cases, the prediction obtained in the symmetric limit is close to what observed, and the symmetry breaking only provides the moderate correction necessary for an accurate description. In other cases, the symmetry itself either does not provide a prediction, or the prediction is far from the observation: even a leading order description of flavour mixing relies on the symmetry breaking effects. Here, we would like to address such a dichotomy by identifying the complete set of flavour models leading to the first case, i.e. a symmetric limit providing a reasonable leading order description of flavour mixing [16].

Let us consider a generic (continuous or discrete, simple or not, abelian or non-abelian, or arbitrary combinations of the above) flavour group $G$. In order to provide a precise formulation of the problem, let us write the charged lepton and neutrino mass matrices $m_{E}$ and $m_{v}$ as

$$
\begin{aligned}
& m_{E}=m_{E}^{(0)}+m_{E}^{(1)} \\
& m_{v}=m_{v}^{(0)}+m_{v}^{(1)},
\end{aligned}
$$

where $m_{E}^{(0)}, m_{v}^{(0)}$ are invariant under $G$, while $m_{E}^{(1)}, m_{v}^{(1)}$ are generated by the sources of symmetry breaking, and vanish in the symmetric limit. We say that the symmetric limit associated to $m_{E}^{(0)}$ and $m_{v}^{(0)}$ provides an approximate description of lepton flavour observables if the neutrino and charged lepton masses and the PMNS matrix are in the form shown in Fig. 1. The tables show that we consider all neutrino mass patterns to be close to the symmetric limit, except when all the masses vanish, in which case there is no prediction for the PMNS matrix. A small (or no) correction is indeed sufficient to obtain a fully realistic mass pattern from each of them. All non-degenerate charged lepton mass patterns are also considered to be close to the symmetric limit. In the case in which two or all the three charged leptons are massive in the symmetric limit, a hierarchy needs to be invoked among the non-zero masses in order for the pattern to be close to the observation. In the light of the fact that all PMNS mixings are measured to be large, except, to some extent, the 13

\begin{tabular}{|c|c|c|}
\hline \multicolumn{3}{|c|}{ Neutrino masses } \\
\hline $\mathrm{NH} / \mathrm{IH}$ & $\left(\begin{array}{lll}a & 0 & 0\end{array}\right)$ & $(0 \mathrm{a} a)$ \\
\hline $\mathrm{NH}$ & (a a a) & $(a b 0)$ \\
\hline $\mathrm{IH}$ & $(a b b)$ & $(a b c)$ \\
\hline
\end{tabular}
angle, we consider both the PMNS patterns shown in the figure to be close to the symmetric limit.
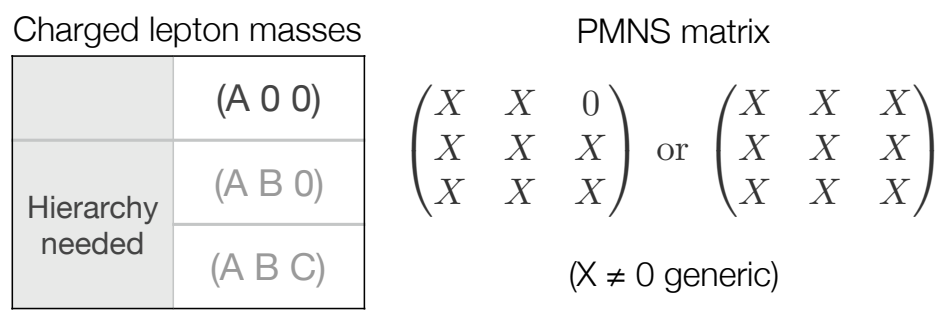

Figure 1: Lepton mass and mixing patterns defined to be close to what observed. The type of neutrino spectrum, normal $(\mathrm{NH})$ or inverted $(\mathrm{IH})$ hierarchical, associated to a given pattern is indicated. A hierarchy among the non-zero parameters is needed in two out the three charged lepton mass patterns.

We aim at providing a full classification of all flavour groups and representations such that the lepton masses and the PMNS matrix are in one of the forms in Fig. 1. We begin by assuming 


\begin{tabular}{|c|c|c|c|}
\hline$U_{l}, U_{e^{c}}$ irreps & masses & $\nu$ hierarchy & PMNS zeros \\
\hline $\begin{array}{lll}1 & 1 & 1\end{array}$ & $(A 00)$ & \multirow{2}{*}{$\mathrm{NH}$ or $\mathrm{IH}$} & \multirow[b]{2}{*}{ none } \\
\hline $1 \quad r \nsupseteq 1$ & $(a b c)$ & & \\
\hline $\begin{array}{lll}1 & 1 & \overline{1}\end{array}$ & $(A 00)$ & \multirow{2}{*}{$\mathrm{IH}$} & \multirow{2}{*}{ none (13) } \\
\hline$\overline{\mathbf{1}} \quad r \nsupseteq \mathbf{1}, \overline{\mathbf{1}}$ & $(0 a a)$ & & \\
\hline $\begin{array}{lll}1 & 1 & 1\end{array}$ & $(A B 0)$ & \multirow{2}{*}{$\mathrm{NH}$ or $\mathrm{IH}$} & \multirow[b]{2}{*}{ none } \\
\hline $1 \quad 1 \quad r \neq 1$ & $(a b c)$ & & \\
\hline $\begin{array}{lll}1 & 1 & 1\end{array}$ & $(A B 0)$ & \multirow{2}{*}{$\mathrm{IH}$} & \multirow{2}{*}{13} \\
\hline$\overline{\mathbf{1}} \quad \overline{\mathbf{1}} \quad r \neq \mathbf{1}$ & $(0 a a)$ & & \\
\hline $\begin{array}{lll}1 & 1 & 1\end{array}$ & $(A B C)$ & \multirow{2}{*}{$\mathrm{NH}$ or $\mathrm{IH}$} & \multirow{2}{*}{ none } \\
\hline 11 & $(a b c)$ & & \\
\hline 1 & $(A B C)$ & \multirow{2}{*}{$\mathrm{IH}$} & \multirow{2}{*}{$\mathbf{1 3}, 23,33$} \\
\hline$\overline{1}$ & $(0 a a)$ & & \\
\hline
\end{tabular}

Table 1: Structure of the irreducible components of the representations $U_{l}, U_{e^{c}}$ providing an approximate description of lepton flavour observables in the symmetric limit. The first column shows the decomposition of $U_{l}$ and $U_{e^{c}}$, one above the other. Boldface fonts denote complex representations, regular fonts denote real representations, with specified dimension. Primes are used to distinguish inequivalent representations. " $r$ " denotes a generic, possibly reducible representation. The second column shows the corresponding pattern of charged lepton and neutrino masses in the symmetric limit, one above the other, and the third column the type of neutrino hierarchy. The PMNS has one or no zeros, and the position of the possible zero is indicated.

that the flavour symmetry constrains the light neutrino Majorana mass matrix $m_{v}$ or, equivalently, the Weinberg operator [17]. Despite the huge (infinite) number of possible groups and representations, the problem can be studied in full generality, and has a simple solution. This is because the pattern of lepton masses and mixings depends on the group and representation only through the dimension, type (real, pseudoreal, complex), and equivalence of the irreducible components of the representations $U_{l}, U_{e^{c}}$ on the lepton doublets $l_{i}$ and charged lepton singlets $e_{i}^{c} \sim \overline{e_{R i}}, i=1,2,3$.

The classes of representations leading to viable patterns, as defined in Fig. 1, are shown in Table 1. There are only six cases. In the second case the presence of a zero in the PMNS matrix depends on the symmetry breaking effects, which determine in that case the 12 mixing angle [1829]. In the last case, the position of the zero depends on the relative size of the $A, B, C$ parameters. In the three cases in which a normal hierarchical neutrino spectrum is allowed, the neutrino mass matrix is totally unconstrained. The representation on the lepton doublets reduces in fact to three equivalent real one-dimensional representations. This means that $U_{l}$ just changes the overall sign of the three lepton doublets, and that any neutrino mass matrix is allowed. The flavour symmetry does not provide any constraint on neutrino masses and mixings (anarchy [30,31]). In the three cases (shaded in Table 1) in which the flavour symmetry does provide an input, the neutrino masses turn out to be inverted hierarchical. Therefore, if the present hint for a normal hierarchy [32-34] were confirmed, we would conclude that an understanding of lepton flavour can only come from symmetry breaking effects. The detailed proofs of such results can be found in [16].

The above conclusion is based on the assumption that the flavour symmetry constrains the light neutrino mass matrix or, equivalently, the Weinberg operator. Let us now assume that the effective Weinberg operator is generated by some "ultraviolet" (UV) physics, and let us consider 
type I seesaw as a prototypical example. Would the same conclusions hold if the flavour symmetry constrained the seesaw lagrangian? The answer is: not necessarily. While all the light neutrino mass matrices obtained from an invariant seesaw lagrangian are also invariant with respect to the low-scale action of the flavour symmetry, not all invariant light neutrino mass matrices can always be obtained from an invariant seesaw lagrangian [35].

There are two reasons why the high-scale and low-scale descriptions can turn out not to be equivalent. The first is that the heavy singlet neutrino mass matrix can be forced to be singular in the symmetric limit. In such a case, at least one singlet neutrino is massless in the symmetric limit. The seesaw formula can then be applied only once symmetry breaking effects have taken into account, thus providing all singlet neutrinos with a (heavy) mass. The condition for the singlet neutrino mass matrix to be allowed to be non-singular in the symmetric limit is that the representation of the flavour group on the neutrino singlets be vectorlike.

Even when the heavy singlet neutrino mass matrix is allowed to be non-singular in the symmetric limit, and the seesaw formula can be flawlessly applied in that limit, the high- and low-scale implementations of the flavour symmetry can still be inequivalent, for a different reason. A necessary and sufficient condition for the equivalence in this case is in fact that the vectorlike component of the representation on lepton doublets be part of the representation on the neutrino singlets.

A proof of the above statements can be found in [35]. Here, it suffices to illustrate the last point with an example. Consider a $U(1)$ flavour symmetry under which the three lepton doublets have charges $(1,0,0)$ and the neutrino singlets have charges $(1,-1,0)$ [36]. The representation on the neutrino singlets is vectorlike, so that the seesaw formula can be applied in the symmetric limit. The Dirac and heavy Majorana neutrino mass matrices, $m_{N}$ (in "RL" convention) and $M$, are constrained to be in the form

$$
m_{N}=\left(\begin{array}{ccc}
0 & 0 & 0 \\
X & 0 & 0 \\
0 & X & X
\end{array}\right), \quad M=\left(\begin{array}{ccc}
0 & X & 0 \\
X & 0 & 0 \\
0 & 0 & X
\end{array}\right),
$$

where no special relation is enforced among the non-vanishing entries denoted by $X$ (except of course $M_{12}=M_{21}$ ). The light neutrino mass matrix obtained from the seesaw mechanism necessarily has rank 1 in the symmetric limit. On the other hand, the low-scale version of the flavour theory, allows neutrino mass matrices with a generic, non-vanishing, rank-two block. We then conclude that not all the invariant light neutrino mass matrices can be obtained from an invariant seesaw lagrangian.

Having established that the low- and high-scale implementations of the same flavour symmetry can be inequivalent, we can revisit the conclusion we obtained within the low-scale implementation of the flavour symmetries. We concluded in that case that a non-trivial understanding of lepton flavour can only come from symmetry breaking effects, if neutrinos are indeed normal hierarchical. This remains true at high-scale in all the cases in which the low- and high-scale implementations are equivalent, and in particular when i) the representation on neutrino singlets is vectorlike and ii) the vectorlike component of the representation on lepton doublets is part of the representation on the neutrino singlets. On the other hand, examples of predictive flavour theories leading to a normal hierarchy in the symmetric limit can be found in high-scale flavour theories, provided that 
high- and low-scale implementations are not equivalent. A more detailed discussion of the viable cases can be found in [35].

\section{References}

[1] C. D. Froggatt and H. B. Nielsen, Hierarchy of Quark Masses, Cabibbo Angles and CP Violation, Nucl. Phys. B147 (1979) 277-298.

[2] J. Bijnens and C. Wetterich, Fermion Masses From Symmetry, Nucl. Phys. B283 (1987) 237-267.

[3] M. Leurer, Y. Nir and N. Seiberg, Mass matrix models, Nucl. Phys. B398 (1993) 319-342, [hep-ph/9212278].

[4] M. Dine, R. G. Leigh and A. Kagan, Flavor symmetries and the problem of squark degeneracy, Phys. Rev. D48 (1993) 4269-4274, [hep-ph/9304299].

[5] L. E. Ibanez and G. G. Ross, Fermion masses and mixing angles from gauge symmetries, Phys. Lett. B332 (1994) 100-110, [hep-ph/9403338].

[6] A. Pomarol and D. Tommasini, Horizontal symmetries for the supersymmetric flavor problem, Nucl. Phys. B466 (1996) 3-24, [hep-ph/9507462].

[7] R. Barbieri, G. R. Dvali and L. J. Hall, Predictions from a U(2) flavor symmetry in supersymmetric theories, Phys. Lett. B377 (1996) 76-82, [hep-ph/9512388].

[8] C. D. Carone, L. J. Hall and H. Murayama, $\left(S_{3}\right)^{3}$ flavor symmetry and $p \rightarrow K^{0} e^{+}$, Phys. Rev. D53 (1996) 6282-6291, [hep-ph/9512399].

[9] E. Dudas, C. Grojean, S. Pokorski and C. A. Savoy, Abelian flavor symmetries in supersymmetric models, Nucl. Phys. B481 (1996) 85-108, [hep-ph/9606383].

[10] R. Barbieri, L. J. Hall, S. Raby and A. Romanino, Unified theories with U(2) flavor symmetry, Nucl. Phys. B493 (1997) 3-26, [hep-ph/9610449].

[11] R. Barbieri, L. J. Hall and A. Romanino, Consequences of a U(2) flavor symmetry, Phys. Lett. B401 (1997) 47-53, [hep-ph/9702315].

[12] C. D. Carone and L. J. Hall, Neutrino physics from a U(2) flavor symmetry, Phys. Rev. D56 (1997) 4198-4206, [hep-ph/9702430].

[13] N. Irges, S. Lavignac and P. Ramond, Predictions from an anomalous U(1) model of Yukawa hierarchies, Phys. Rev. D58 (1998) 035003, [hep-ph/ 9802334 ].

[14] J. K. Elwood, N. Irges and P. Ramond, Family symmetry and neutrino mixing, Phys. Rev. Lett. 81 (1998) 5064-5067, [hep-ph/9807228].

[15] L. Ferretti, S. F. King and A. Romanino, Flavour from accidental symmetries, JHEP 11 (2006) 078, [hep-ph/0609047].

[16] Y. Reyimuaji and A. Romanino, Can an unbroken flavour symmetry provide an approximate description of lepton masses and mixing?, JHEP 03 (2018) 067, [1801.10530].

[17] S. Weinberg, Baryon and Lepton Nonconserving Processes, Phys. Rev. Lett. 43 (1979) 1566-1570.

[18] P. H. Frampton, S. T. Petcov and W. Rodejohann, On deviations from bimaximal neutrino mixing, Nucl. Phys. B687 (2004) 31-54, [hep-ph/ 0401206 ].

[19] A. Romanino, Charged lepton contributions to the solar neutrino mixing and $\theta_{13}$, Phys. Rev. D70 (2004) 013003, [hep-ph/ 0402258$].$ 
[20] S. F. King, Predicting neutrino parameters from SO(3) family symmetry and quark-lepton unification, JHEP 08 (2005) 105, [hep-ph/ 0506297 ].

[21] S. Antusch and S. F. King, Charged lepton corrections to neutrino mixing angles and CP phases revisited, Phys. Lett. B631 (2005) 42-47, [hep-ph/ 0508044 ].

[22] K. A. Hochmuth, S. T. Petcov and W. Rodejohann, $U_{P M N S}=U_{l}^{\dagger} U_{v}$, Phys. Lett. B654 (2007) 177-188, [0706.2975].

[23] P. S. Bhupal Dev, R. N. Mohapatra and M. Severson, Neutrino Mixings in SO(10) with Type II Seesaw and $\theta_{13}$, Phys. Rev. D84 (2011) 053005, [1107.2378].

[24] S. Dev, S. Gupta and R. Raman Gautam, Parametrizing the Lepton Mixing Matrix in terms of Charged Lepton Corrections, Phys. Lett. B704 (2011) 527-533, [1107.1125].

[25] D. Marzocca, S. T. Petcov, A. Romanino and M. Spinrath, Sizeable $\theta_{13}$ from the Charged Lepton Sector in SU(5), (Tri-)Bimaximal Neutrino Mixing and Dirac CP Violation, JHEP 11 (2011) 009, [1108.0614].

[26] G. Altarelli, F. Feruglio and L. Merlo, Tri-Bimaximal Neutrino Mixing and Discrete Flavour Symmetries, Fortsch. Phys. 61 (2013) 507-534, [1205.5133].

[27] D. Marzocca, S. T. Petcov, A. Romanino and M. C. Sevilla, Nonzero $\left|U_{e 3}\right|$ from Charged Lepton Corrections and the Atmospheric Neutrino Mixing Angle, JHEP 05 (2013) 073, [1302 . 0423 ].

[28] S. Gollu, K. N. Deepthi and R. Mohanta, Charged lepton correction to tribimaximal lepton mixing and its implications to neutrino phenomenology, Mod. Phys. Lett. A28 (2013) 1350131, [1303. 3393].

[29] D. Marzocca and A. Romanino, Stable fermion mass matrices and the charged lepton contribution to neutrino mixing, JHEP 11 (2014) 159, [1409.3760].

[30] L. J. Hall, H. Murayama and N. Weiner, Neutrino mass anarchy, Phys. Rev. Lett. 84 (2000) 2572-2575, [hep-ph/9911341].

[31] N. Haba and H. Murayama, Anarchy and hierarchy, Phys. Rev. D63 (2001) 053010, [hep-ph/0009174].

[32] F. Simpson, R. Jimenez, C. Pena-Garay and L. Verde, Strong Bayesian Evidence for the Normal Neutrino Hierarchy, JCAP 1706 (2017) 029, [1703.03425].

[33] F. Capozzi, E. Di Valentino, E. Lisi, A. Marrone, A. Melchiorri and A. Palazzo, Global constraints on absolute neutrino masses and their ordering, Phys. Rev. D95 (2017) 096014, [1703. 04471$].$

[34] P. F. de Salas, D. V. Forero, C. A. Ternes, M. Tortola and J. W. F. Valle, Status of neutrino oscillations 2017, 1708.01186.

[35] Y. Reyimuaji and A. Romanino, in preparation.

[36] G. Altarelli and F. Feruglio, Models of neutrino masses from oscillations with maximal mixing, JHEP 11 (1998) 021, [hep-ph/9809596]. 